\title{
Significados y prácticas de consumo conspicuo asociados a la posesión de perros criollos o de raza
}

\author{
Lorena Ballén Molina; Claudia Pineda-Marín; Marithza Sandoval-Escobar; Claudia Mercedes Padrón Mercado
}

Cómo citar este artículo:

Ballén Molina, L., Pineda-Marín, C., Sandoval-Escobar, M., \& Padrón Mercado, C. M. (2021). Significados y prácticas de consumo conspicuo asociados a la posesión de perros criollos o de raza. Acta Colombiana de Psicología, 24(1), 141153. https://doi.org/10.14718/ACP.2021.24.1.13

Recibido, agosto 31/2020; Concepto de evaluación, octubre 28/2020; Aceptado, noviembre 09/2020

\author{
Lorena Ballén Molina ${ }^{1}$ \\ ORCID: https://orcid.org/0000-0001-7063-4787 \\ Fundación Universitaria Konrad Lorenz, Bogotá, Colombia. \\ Claudia Pineda-Marín \\ ORCID: https://orcid.org/0000-0002-0952-6522 \\ Fundación Universitaria Konrad Lorenz, Bogotá, Colombia. \\ Marithza Sandoval-Escobar \\ ORCID: https://orcid.org/0000-0003-1938-0675 \\ Fundación Universitaria Konrad Lorenz, Bogotá, Colombia. \\ Claudia Mercedes Padrón Mercado \\ ORCID: https://orcid.org/0000-0003-2717-0344 \\ Fundación Universitaria Konrad Lorenz, Bogotá, Colombia.
}

\begin{abstract}
Resumen
Esta investigación tuvo como objetivo identificar las representaciones sociales y las prácticas de consumo conspicuo en propietarios de perros en Colombia. Por tanto, se realizó un estudio mixto en una muestra de 96 hombres y mujeres mayores de 18 años, con, primero, una fase cualitativa en la que se exploraron las asociaciones dadas a las representaciones sociales de perros criollos y de raza por parte de propietarios de mascotas y se abordaron los significados atribuidos a las prácticas de consumo conspicuo; y, segundo, una fase cuantitativa en la que se hicieron análisis descriptivos, con lo cual se confirmó la estructura de las representaciones sociales. Como resultados se encontró que se reconoce a los perros criollos y de raza con alto valor afectivo, y que el perro criollo se asocia a condiciones de vulnerabilidad y se reconoce como perro "todo terreno", mientras que el perro de raza involucra cuidado e inversión económica y se identifica como un perro "visible" en la sociedad. Al final se discute respecto a cómo las representaciones sociales acerca de la tenencia de un perro, además del valor afectivo, involucran actividades exhibitorias, de demostración social y, a su vez, un estatus social y moral.

Palabras clave: representaciones sociales, perro, criollo, raza, consumo conspicuo.
\end{abstract}

\section{Meanings and practices of conspicuous consumption associated with the possession of creole or pedigree dogs}

\begin{abstract}
This research aimed to identify social representations and conspicuous consumption practices in dog owners in the city of Bogotá, Colombia. The sample included 96 men and women over the age of 18. A mixed study was carried out including first, a qualitative phase in which the social representations of dogs' owners - both creole and breed- were explored, as well as the meanings attributed to conspicuous consumption practices. Secondly, in the quantitative phase, descriptive analyses
\end{abstract}

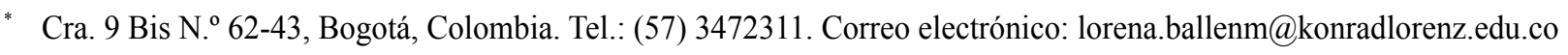


were carried out and thus the structure of social representations was confirmed. The results showed that the affective value of both the creole and breed dogs was acknowledged and that the creole dog is associated with conditions of vulnerability and regarded as an all-terrain dog, whereas breed dogs imply care and economic investment and are identified as "visible" dogs in society. It is discussed how social representations about dog ownership, in addition to the affective value, involve exhibition activities, social demonstration, and in turn, social and moral status.

Keywords: social representations, dog, creole, breed, conspicuous consumption.

\section{Introducción}

El perro como animal doméstico y de compañía cobra gran valor afectivo en las personas que los poseen, y las conductas de consumo que rodean esta relación se constituyen como un tema social interesante de indagar. En temas de consumo, por ejemplo, tener empatía hacia los animales puede tener consecuencias inmediatas en la conducta de los potenciales consumidores, como es el caso de las personas que han abolido la compra e ingesta de productos derivados de los animales (Camilleri et al., 2020).

De hecho, según el estudio de World Animal Protection (2018) realizado en Latinoamérica con más de diez mil propietarios de perros, los propietarios consideran a sus mascotas como "hijos" o, al menos, como parte de la familia, lo que evidencia una nueva dinámica social que, de acuerdo con Cabella y Nathan (2018), está relacionada con la disminución de la fecundidad en América Latina y el Caribe. Incluso, según Coll y De La Rosa (2018), este interés y participación de los perros en la vida de las personas va de la mano con cambios en materia legislativa, sobre todo en lo relacionado con los derechos de los animales y su dignificación como seres que sienten, al igual que el ser humano.

En cuanto a las características del mercado, los resultados de Euromonitor (citado por Coll \& De La Rosa, 2018) demuestran que la industria de mascotas en Colombia en el año 2017 creció en un $13 \%$, ocupando el cuarto puesto en América Latina después de Brasil, México y Chile, y que las categorías que han impulsado esta industria son el alimento y el cuidado para la mascota, junto con servicios de lujo para mascotas, como los servicios de cuidado y belleza. De igual manera, Ballestas (2018) identificó en su estudio que el mercado de las mascotas crece por encima de la inflación, pues, por ejemplo, en 2017 estuvo casi cuatro veces por encima del índice de precios al consumidor (IPC) — que fue de $4.09 \%$ - y que en 4 de cada 10 familias hay al menos una mascota, en la cual, de hecho, se tiene un gasto promedio en la alimentación de la mascota de alrededor de 180 dólares.

Ahora bien, dada las transformaciones sociales y culturales que afectan la relación entre los humanos y los animales de compañía, además de la incidencia de dicha relación en las prácticas de consumo, en el presente estudio se indagó respecto a las representaciones sociales y las prácticas de consumo conspicuo de tenedores de perros criollos y de raza.

En particular, para este trabajo se abordaron dos categorías: (a) el consumo conspicuo y (b) las representaciones sociales -entendidos como conjuntos de significados construidos socialmente. Con respecto al consumo conspicuo, este ha sido definido como un medio para entender la imagen idealizada del individuo y de cómo quiere que otros lo reconozcan, y como la forma en que se clasifican los individuos —en términos individuales y grupales - sobre la base de las cosas que hacen y cómo eligen invertir su dinero (Onofre-Chaves, 2017).

En el inicio de la investigación contemporánea sobre el consumo conspicuo, Veblen (1899) estudió estos hábitos de consumo y los definió como un medio de distinción valorativa entre individuos que se asocia al estatus o dominio puro, con características de ostentación, estética, exhibición, estatus y poder económico dentro una sociedad. Desde entonces, los diferentes estudios del consumo destacan su importancia en la compra de las marcas, así como el significado de estas para la percepción del estatus y la construcción de la marca (Sandoval-Escobar et al., 2018).

En particular, Vigneron y Johnson (1999) describieron inicialmente cinco valores diferenciales entre marcas de prestigio y marcas de no prestigio, que dependen de un marco socioeconómico particular, y estos son: (a) el valor visible percibido, que se relaciona con el consumo de marcas de prestigio, que es visto como una señal de riqueza y estatus; (b) el valor único percibido, que se entiende como el caso en el que, al poseer una marca en particular, la persona se considera prestigiosa; (c) el valor social percibido, que hace referencia a aquellos aspectos del juego de roles y el valor social de las marcas de prestigio que pueden ser fundamentales en la decisión de comprar; (d) el valor hedónico percibido, que está relacionado con la satisfacción de deseos emocionales por medio de marcas prestigiosas, donde se identifican los beneficios intangibles subjetivos de un producto y el atractivo estético como determinantes en la selección de la marca; y, finalmente, (d) el valor de calidad percibido, que se entiende como el prestigio que el consumidor otorga a una marca por la superioridad técnica y el cuidado durante el proceso de producción. 
Por otra parte, Vigneron y Johnson (2004) identificaron que los profesionales ven el lujo como un factor importante para diferenciar una marca en una categoría de producto, así como un motor central de preferencia del consumidor, y como un denominador de uso entre culturas. Para estos autores, la percepción de lo que es y no es una marca de lujo puede depender del contexto y de las personas involucradas.

Ahora bien, con respecto a la diferenciación entre marcas de prestigio y aquellas que no lo son, se han identificado relaciones importantes con aspectos ideológicos y sociodemográficos. Otterbring et al. (2020), por ejemplo, proponen un modelo con prestigio y dominio como vías duales para lograr estatus social; mientras que Trigg (2016), por otra parte, confirmó la asociación de las marcas con la jerarquía social, y esta con patrones de dominio que aseguran posiciones particulares en la jerarquía social. En este contexto, Figueras y Moreno (2013) encontraron que la estimación social e individual se determina por el tipo y el nivel de gasto visible en el consumo, y que, a su vez, el tipo de gasto aceptado en la sociedad o clase a la que pertenece el comprador permite determinar, en parte, cuál ha de ser su nivel de vida.

Como se observa, existe una relación entre el consumo conspicuo y el lujo, y aunque los dos conceptos son difíciles de diferenciar (Kiattipoom \& Heesup, 2019; Ruiz, 2018), es posible identificar que el lujo se relaciona con la ostentación que genera reconocimiento social, distinción y envidia, y que está asociado con todo aquello que posee experiencias únicas - en términos de consumo experiencial-. En ese mismo sentido, la condición social se configura, en el lujo, como un rasgo característico según el tipo de práctica de consumo que se ejecute y los elementos que la conforman, y que, por tanto, transmite significado simbólico entre los grupos.

Por su parte, el consumo conspicuo se relaciona con el consumo visible, cuyos significados pueden estar relacionados con el estatus, pero también con otros aspectos de la vida social de las personas (Mozzocco et al., 2012). Particularmente, para este estudio se considera importante observar las prácticas de consumo conspicuo, porque la tenencia de perros implica una exhibición pública en espacios sociales en donde los dueños interactúan y muestran parte de su estilo de vida a través de los perros, así como también desarrollan una dinámica privada en el entrenamiento y el trato cotidiano (Barrera et al., 2012; Jakovcevic et al., 2011).

Ahora bien, otro de los conceptos que cobra vital importancia para la investigación es el de las representaciones sociales, que son una propuesta teórica en la cual se esboza un planteamiento metodológico dentro del análisis del sentido común y de lo cotidiano, y que se puede valorar como una explicación importante en el estudio de la construcción social de la realidad (Materán, 2008). Analizando los aspectos asociados con los significados simbólicos, se encuentra que los grupos sociales desarrollan un conjunto de significados compartidos que brindan un conjunto de marcos de interpretación.

Específicamente, en lo que se ha denominado teoría de las representaciones sociales (Molina et al., 2010; Navarro \& Restrepo, 2013; Vergara, 2008), las representaciones sociales son vistas como el resultado de la relación entre un objeto de representación - algo, alguien, o un evento- y un sujeto que lo representa - individuos o grupos - dentro de un marco histórico y cultural de referencia. No obstante, de acuerdo con Quiroz (2004), existen diferentes perspectivas sobre las representaciones sociales, donde destacan dos grandes corrientes, denominadas clásica-figurativa y estructuralista (Abric, 2001; Moscovici, 1985).

En particular, Abric (2001) plantea que las representaciones están configuradas por un núcleo central y un sistema periférico. El núcleo central es la parte estable de la representación social, y su función es determinar los significados y la organización de los contenidos; además, su aspecto normativo se relaciona con las dimensiones socioafectiva e ideológica, que tienen que ver con las convicciones de los sujetos y con la estabilidad del comportamiento basado en la representación - no obstante, el núcleo central es vulnerable a la influencia que proviene del sistema periférico, por tanto, está en un proceso de cambio constante$\mathrm{Y}$, por otra parte, los elementos periféricos se organizan alrededor del núcleo central y se relacionan directamente con él, y abarcan información y juicios formulados en relación con un objeto y su entorno. Estos elementos están jerarquizados según la cercanía al núcleo central, pues los que se encuentran próximos al núcleo desempeñan un papel importante en la concreción del significado de la representación, mientras que los más distantes ilustran, aclaran y justifican esta significación. Asimismo, desde esta perspectiva las representaciones poseen las funciones de saber, identidad, orientación y justificación, las cuales, a su vez, permiten la diferenciación social y el desarrollo de prácticas sociales (Abric, 2001).

Respecto al caso que nos ocupa, la relación entre humanos y perros fue transformándose, debido a que se pasó de un vínculo inicial centrado en el trabajo - p. ej., el perro de caza-, a uno en el que los animales fueron más de compañía, y en donde su función pasó tanto a la protección de un lugar como a la ostentación y la señalización de la posición social del propietario. En ese contexto, la tenencia de perros como mascota ha pasado de un vínculo estrictamente funcional al hecho de constituir relaciones estrechas tanto internas al individuo - grupo tenedor - como en términos de la comunicación social. 
144

Teniendo esto en cuenta, Power (2011) describió la manera en que los perros lograron incorporarse a las familias, y planteó la noción de una familia humano-perro o "familia más que humana", donde se encuentran al menos tres formas en la que los perros se convirtieron en familia - específicamente, de un grupo de personas entrevistadas entre los años 2007 y 2008-: en primer lugar, el grupo reconoció a los perros como "niños peludos", en segundo lugar, como parte de la relación familiar, al involucrarlos en sus diálogos, $\mathrm{y}$, en tercer lugar, la acción individual de los perros fue reconocida como la conformación de la familia y el hogar.

Finalmente, en cuanto al valor económico y significado visible a partir de las mascotas, Kirk (2019) encontró que los consumidores les adjudican una alta valoración económica a los perros, más que a los gatos, lo cual se evidencia por la propensión que muestran a pagar más por cirugías para salvar sus vidas, así como por asumir gastos veterinarios y productos costosos para mascotas, por lo que se puede deducir no solo un involucramiento emocional con la mascota, sino un sentido económico en la posesión.

A pesar de estos hallazgos, aún no son claras las relaciones entre la valoración económica y los significados emocionales que poseen las mascotas para los propietarios, particularmente en el caso de perros cuyas razas son costosas y exclusivas — perros de raza—, en comparación con aquellos a los que no se asocia dicha exclusividad - perros criollos-. Identificar estas diferencias es importante para explicar la manera en que los significados varían y modifican la construcción social de la familia, sobre todo porque el perro llega a ser considerado parte de la familia, y porque, al parecer, este le otorga un sentido de estatus y de identidad personal a quien posee la mascota.

Teniendo lo anterior en cuenta, la presente investigación tuvo el objetivo de identificar las representaciones sociales respecto a la tenencia de perros en el hogar, diferenciando los significados asociados a los perros de raza y los perros criollos, e identificando las prácticas de consumo conspicuo asociadas a ellos.

\section{Método}

\section{Tipo de estudio y diseño}

El presente estudio tuvo un diseño mixto exploratorio, secuencial y derivativo (Folgueiras-Bertomeu \& Ramirez, 2017), que se dividió en dos fases: en la primera, que tuvo un carácter cualitativo, se realizó un estudio — con el enfoque del interaccionismo simbólico-de los procesos mediante los cuales se desarrollaron los significados de la raza en propietarios de perros, donde se identificaron las representaciones sociales -núcleo central y sistemas periféricos-y las prácticas de consumo conspicuo de los propietarios de mascotas; y en la segunda fase, de carácter cuantitativo, se empleó un diseño descriptivo multidimensional que buscó confirmar la estructura de la representación social — desde el enfoque propuesto por Abric (2001) - a partir de un análisis lexicométrico con conteo de frecuencias de palabras jerarquizadas según las descripciones de los participantes en torno a las expresiones acerca de los perros criollos y los perros de raza (Blasco et al., 2020).

\section{Participantes}

Se contó con la participación de 96 personas, hombres y mujeres, propietarias de perros, y residentes en la ciudad de Bogotá, Colombia. Específicamente, en la primera fase - cualitativa - participaron 18 personas, mientras que en la segunda - cuantitativa - lo hicieron 78 personas.

En la segunda fase se identificó que el $62 \%$ era de sexo femenino y el $38 \%$ de sexo masculino, con una edad de 32 años en promedio; $y$, en resumen, la muestra estuvo en mayor parte conformada por personas sin hijos $(63 \%)$, con formación universitaria $(54 \%)$, solteros $(63 \%)$, con trabajo (60\%), de niveles socioeconómicos bajo (35\%) y medio (45\%), la mayoría de los participantes $(57 \%)$ había tenido un perro durante los últimos dos años, el $81 \%$ indicó que los perros le han gustado toda la vida, y, en lo relacionado con la procedencia de la mascota, el $42 \%$ reportó tener un perro criollo, y el $57 \%$, un perro de raza.

\section{Técnicas e instrumentos}

En la fase cualitativa se desarrollaron entrevistas semiestructuradas a profundidad con una guía como instrumento en donde se construyeron preguntas relacionadas con las categorías que orientaron la investigación. Dicha guía fue evaluada por jueces en aspectos como pertinencia, redacción y coherencia. Adicionalmente, las entrevistas se complementaron con observaciones participantes en donde, a partir de las matrices construidas y evaluadas por jueces, se dejaron registros fotográficos y comentarios de cada lugar visitado.

Por otra parte, en la fase cuantitativa se diseñó un cuestionario de preguntas abiertas y cerradas que permitió confirmar la centralidad y periferia de las representaciones sociales. En particular, este instrumento evaluó las asociaciones de los rasgos de los perros con criterios de estatus y de evaluación social según la raza de la mascota. En segundo lugar, se tomó la adaptación del instrumento validado por Cekavicius y Pajarskaite (2012) para medir consumo conspicuo. En el estudio original, los autores trabajaron con estudiantes hombres y mujeres de edades 
entre los 18 y los 35 años, residentes de EE. UU., y cuyas preguntas fueron adaptadas en lengua y cultura a nuestro contexto; adicionalmente, se hizo un análisis de fiabilidad con alfa de Cronbach, cuyo puntaje fue de .9. Además, el instrumento incluyó escalas con opciones de respuesta tipo Likert de cinco grados ordinales; y, sumado a esto, se incluyeron preguntas abiertas para complementar el análisis de resultados. La pregunta filtro indagó acerca de la tenencia de un perro, la raza de este y cuánto tiempo ha tenido perro.

\section{Procedimiento}

Las entrevistas de la fase cualitativa se desarrollaron en el lugar de domicilio de los participantes, donde se solicitó inicialmente la firma de consentimiento informado y se abordaron posteriormente las preguntas de la guía de entrevista. Las entrevistas fueron grabadas en audio para su posterior transcripción, y a partir de las transcripciones se realizó el proceso de codificación abierta, axial y selectiva en el programa Atlas.ti 7. Por otra parte, los textos también fueron analizados a través de un software de minería de datos, denominado $\mathrm{KH}$ Coder, el cual permite el desarrollo de estadísticas respecto a los datos textuales (Blasco-Gil et al., 2020; Higuchi, 2016).

En cuanto a las observaciones, se visitaron hipermercados y tiendas de barrio especializadas en la comercialización de productos y servicios para mascotas, así como también lugares donde se comercializan perros. Para esto último, se registró lo observado en una matriz y se generó un registro fotográfico de cada lugar.

La segunda fase — cuantitativa — se llevó a cabo por medio de la aplicación del instrumento de manera virtual, el cual tuvo una validación cognitiva mediante de la aplicación piloto a 10 personas que permitió mejorar la redacción de las preguntas. Se enviaron los enlaces para responder las encuestas, en las que se pidió a las personas interesadas que aceptaran y firmaran el consentimiento informado antes de diligenciar el instrumento.

\section{Consideraciones éticas}

La presente investigación se realizó adoptando los lineamientos, consideraciones éticas y criterios legales establecidos al momento de tener interacción con personas participantes en un proceso de investigación, así como habiendo tenido la aprobación previa del comité curricular al que están inscritas las investigadoras. Por lo anterior, el presente estudio se acoge los artículos y títulos que conforman las normas vigentes en Colombia que regulan la investigación en psicología, como lo es la ley 1090 de 2006, en donde se reglamenta el ejercicio de la profesión y se dicta el código bioético y otras disposiciones asociadas con los derechos y protección hacia los participantes de un estudio.

\section{Resultados}

A partir de las técnicas de recolección de información se pudo establecer que los perros poseen una gran cantidad de significados afectivos asociados. Por ejemplo, se encontró que las personas les otorgan roles como "hijos", "parte de la familia" y "canalizadores de energía", entre otros; y algunas de las expresiones presentadas en la Figura 1 indican que, además de su rol familiar, se caracterizan con rasgos de lealtad y de bondad.

De igual modo, a partir de estos datos se identificó la estructura de las representaciones sociales por jerarquía y valor expresivo, donde la palabra "amor" se aparece como núcleo central, seguida, en orden de jerarquía y frecuencia para los participantes, por las palabras "lealtad" y "fidelidad". En el sistema periférico, se encontraron palabras como "compañía", "cuidado", "responsabilidad", "familia", "amigo"y "amistad".

Por otra parte, el análisis del registro fotográfico mostró que en diversos espacios de compra las familias asisten a las tiendas con el perro y desarrollan procesos de compra, lo que requiere el acuerdo familiar y que se asocia con las prácticas observadas en el caso de niños y niñas.

Ahora bien, en cuanto a las asociaciones con el "perro criollo", los participantes se inclinaron hacia un gran reconocimiento de afecto, y se identificaron expresiones como "fuerte", "bonito", "abandono", "calle", "inteligentes" y "fieles", entre otros significados. De hecho, las narrativas se dirigen a caracterizar al perro criollo como alguien que ha tenido experiencias de calle, y por ello se menciona su fortaleza, agradecimiento, protección y necesidad de afecto (véase Figura 2).

En la fase cuantitativa, en cuanto a la estructura por jerarquía y valor expresivo, se identificó la palabra "amor" como núcleo central, y las expresiones que la siguieron en orden de jerarquía y frecuencia fueron "lealtad" y "agradecimiento"; en el sistema periférico, se encontraron las palabras "fidelidad", "compañía", "responsabilidad", "cuidado", "adopción" y "cariño", de modo que, con este lenguaje, los individuos construyen el contexto social y los significados atribuidos a los perros criollos.

Por otra parte, los significados asociados a los "perros de raza" se construyen con factores emocionales y de cuidado hacia la mascota, donde aparecen rasgos asociados con la raza del perro, los cuidados que deben seguirse, el costo de la alimentación y el mantenimiento, entre otras (véase Figura 3). En la fase cuantitativa, en lo expresado por los participantes en cuanto a la estructura por jerarquía y valor expresivo acerca de las representaciones sociales respecto a los perros de raza, se encuentra, igualmente, la palabra "amor" como el núcleo central, seguida, en términos de frecuencia, de las expresiones "cuidado" y "fidelidad". Las 


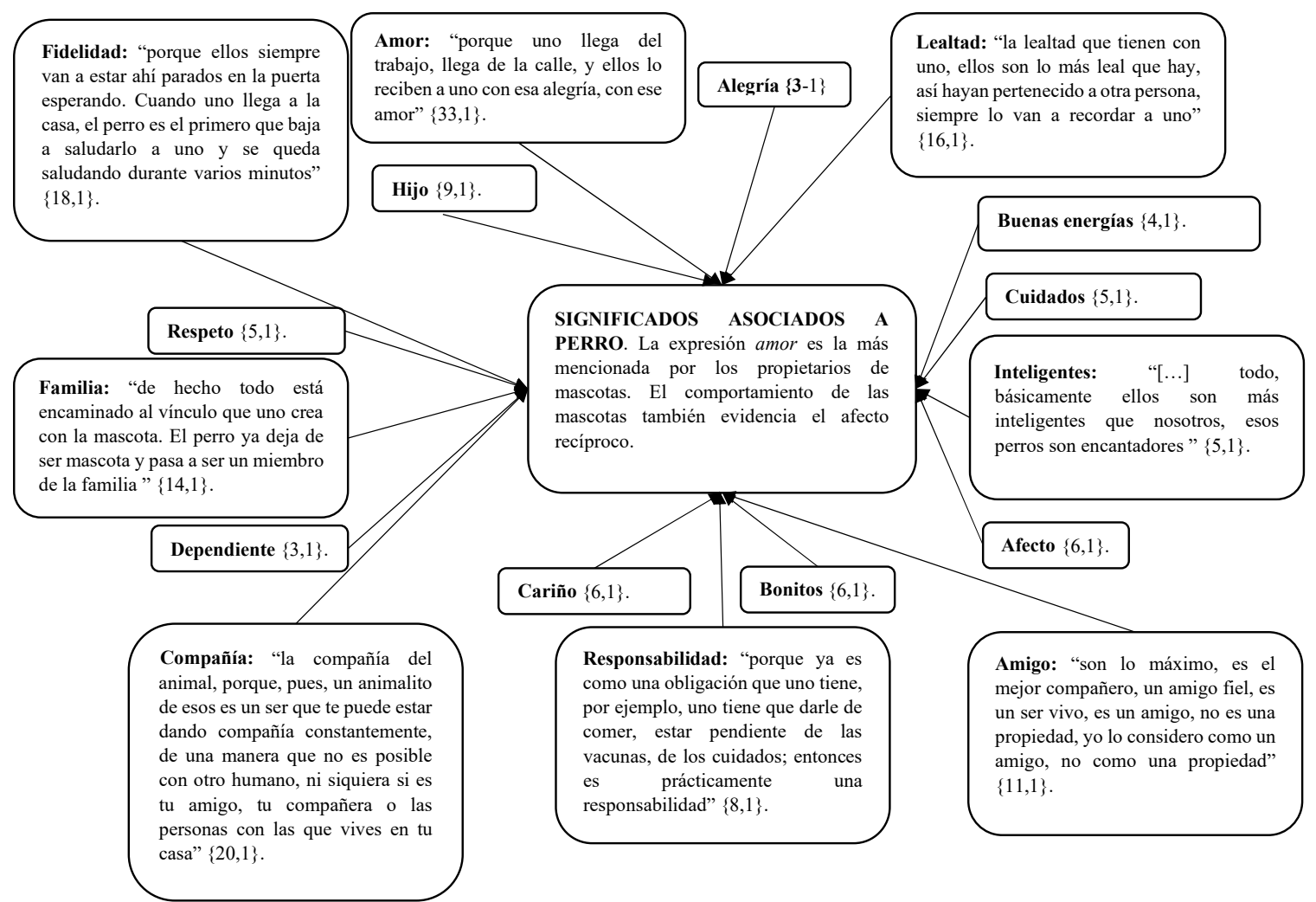

Figura 1.

Red semántica de las representaciones sociales respecto al perro

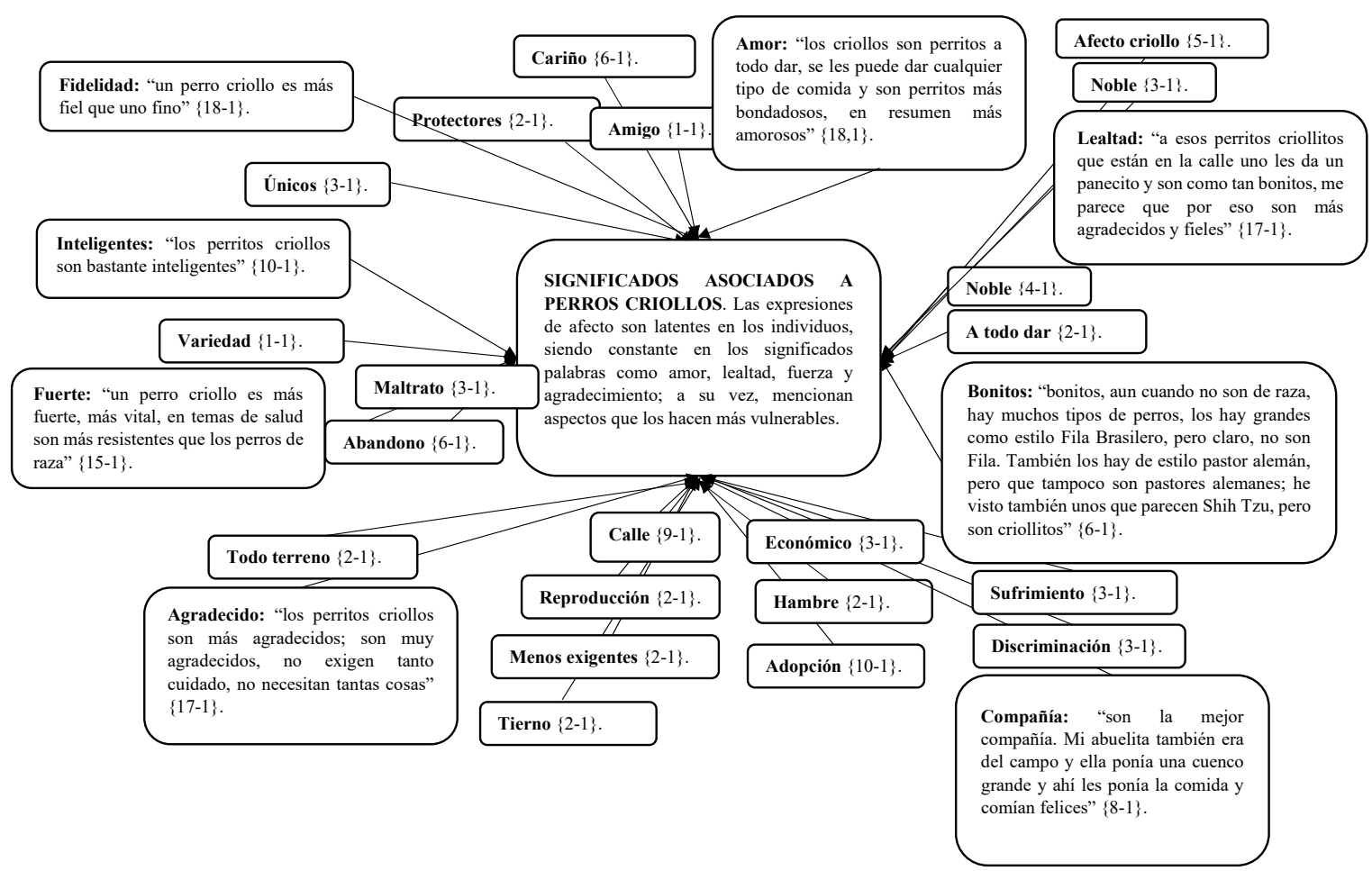

Figura 2.

Red semántica de las representaciones sociales respecto a los perros criollos 
palabras que continúan son, en orden: "lealtad", "compañía", "belleza", "amigo", "compra" y "costo".

Ahora bien, en lo que respecta a los significados asociados a la adquisición de un perro de raza versus un perro criollo, los datos hallados evidencian algunas diferencias. Por ejemplo, como aparece en las figuras 4 y 5 , los participantes consideran que la adquisición de un perro criollo se asocia a personas que cultivan un gusto propio por el perro sin importar su condición y que tienen alta sensibilidad, mientras que, al indagar los factores que motivan la adquisición de un perro de raza, se identificó la asociación con palabras como "dinero", "estatus", "exhibición" y "gustos

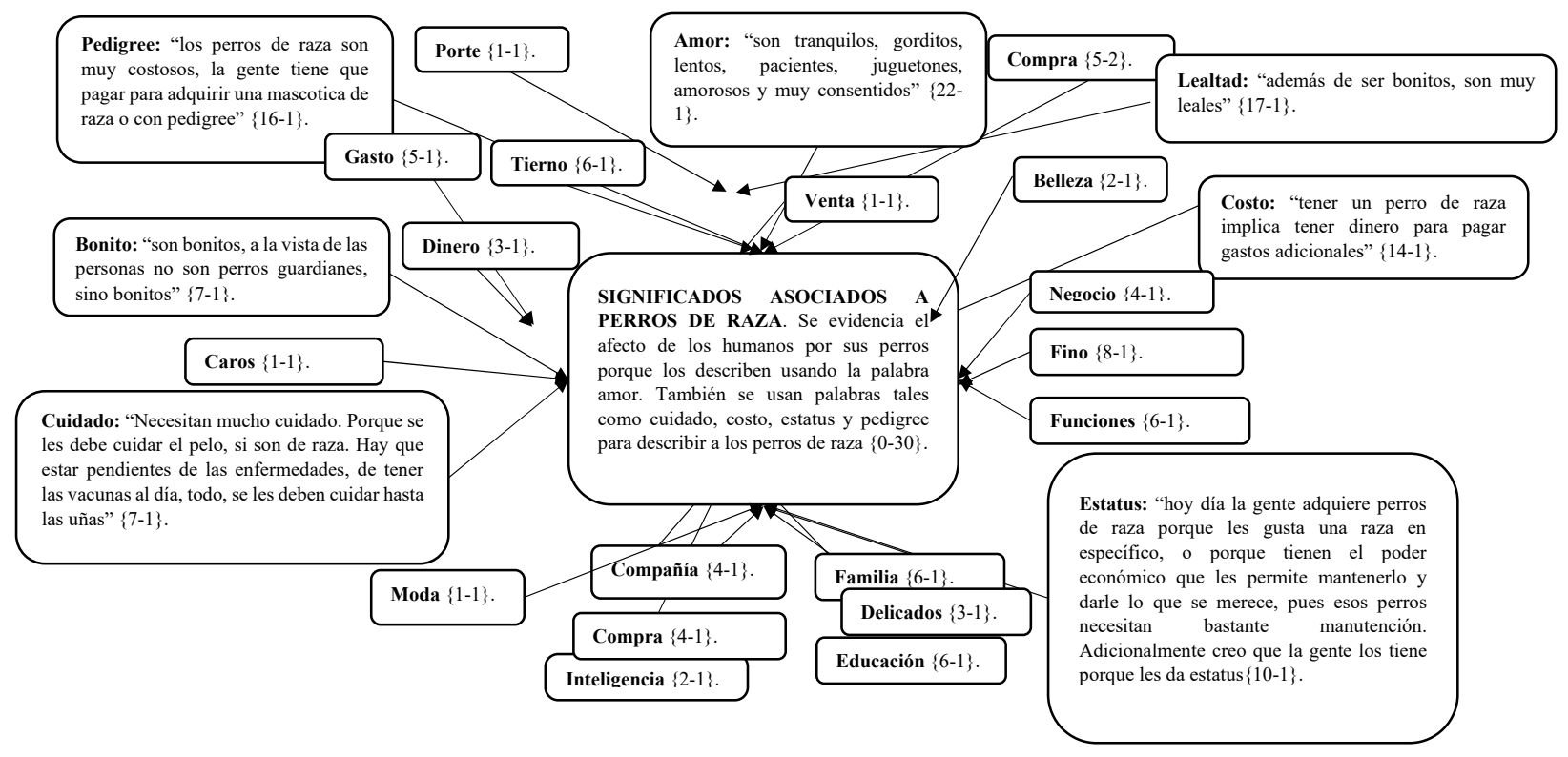

Figura 3.

Red semántica de las representaciones sociales respecto a los perros de raza

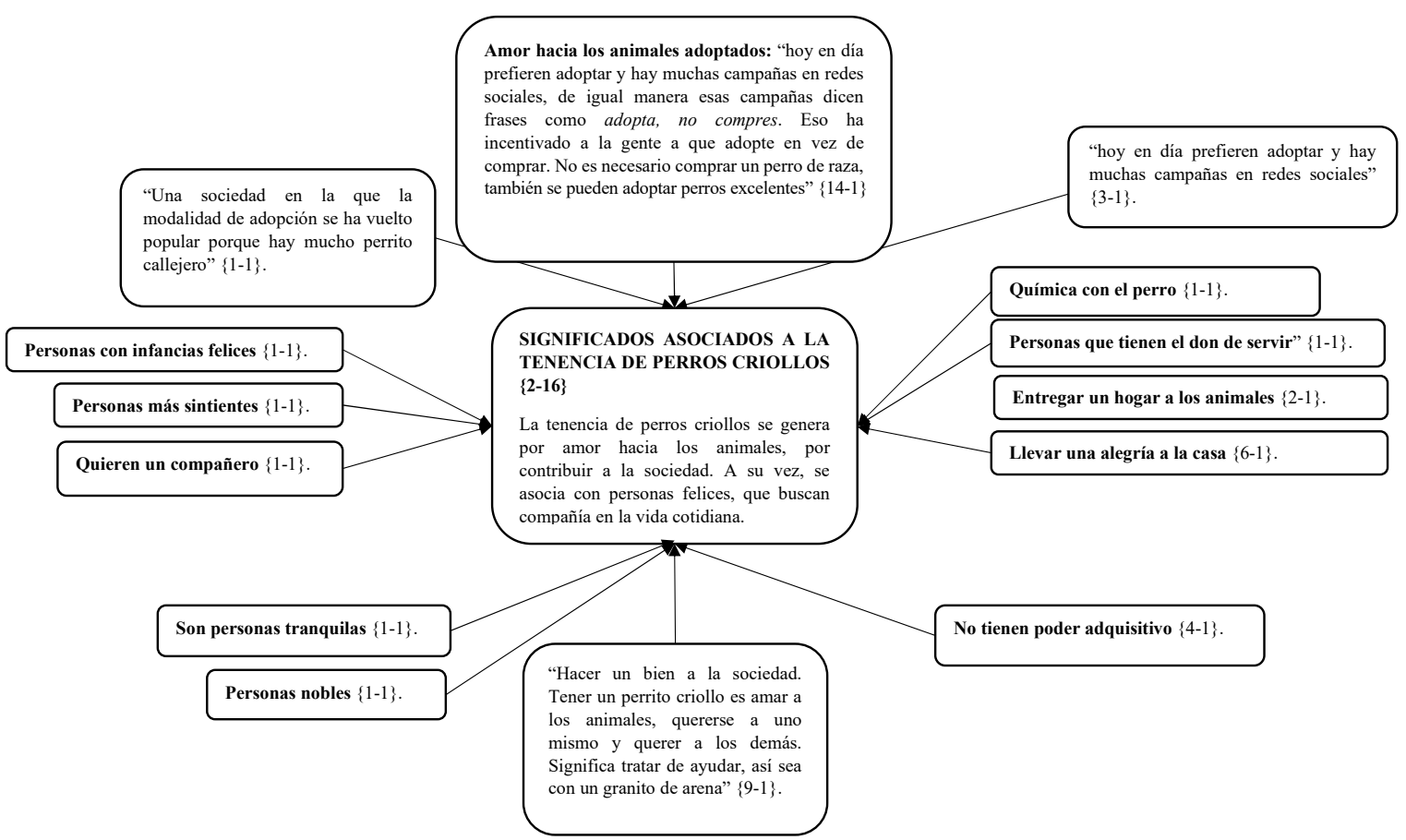

Figura 4.

Red semántica de significados asociados a la tenencia de un perro criollo 


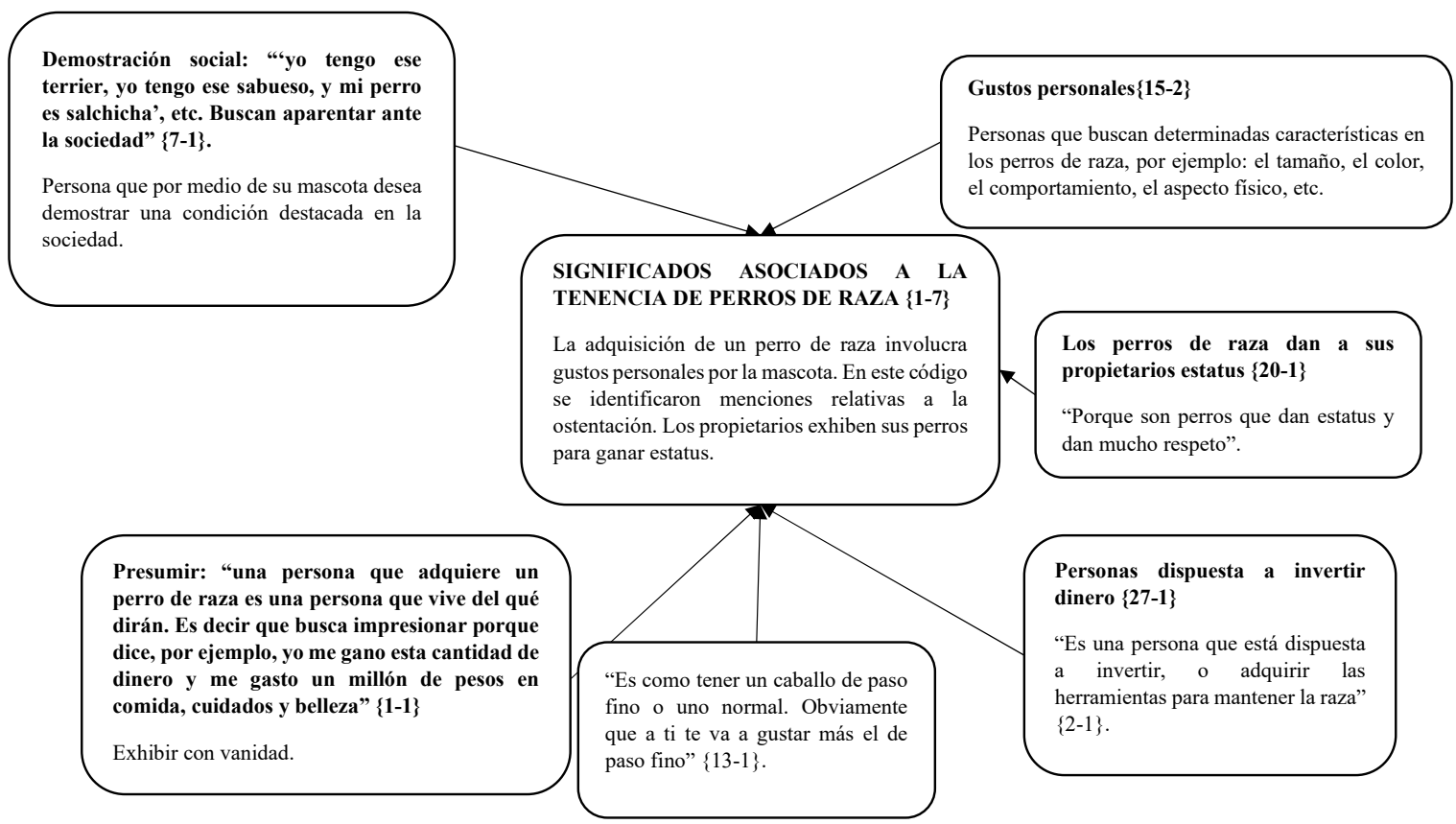

Figura 5.

Red semántica de significados asociados a la tenencia de un perro de raza

personales". En general, las diferencias en los significados muestran que los relacionados con los perros de raza se asocian a un consumo de lujo y visible, mientras que los relacionados con los perros criollos lo hacen con aspectos más funcionales y emocionales.

Más adelante, siguiendo con la fase del análisis cualitativo, se efectuó un conteo de frecuencias para conocer las palabras que mejor definían a los perros de raza y criollos (véase Figura 6), donde se encontró que palabras como "belleza", "compra”, "dinero", “costo", "pedigree", "estatus" y "criadero" fueron más propias de los relatos dados a la tenencia de perros de raza, mientras que palabras como "agradecido", "adopción", "familia", "cariño", "soledad" e "igual" se asociaron en mayor medida a los relatos dados respecto a los perros criollos. Interesantemente, se observa que palabras como "cuidado" aparecen con mayor frecuencia en las descripciones de perros de raza que en los criollos.
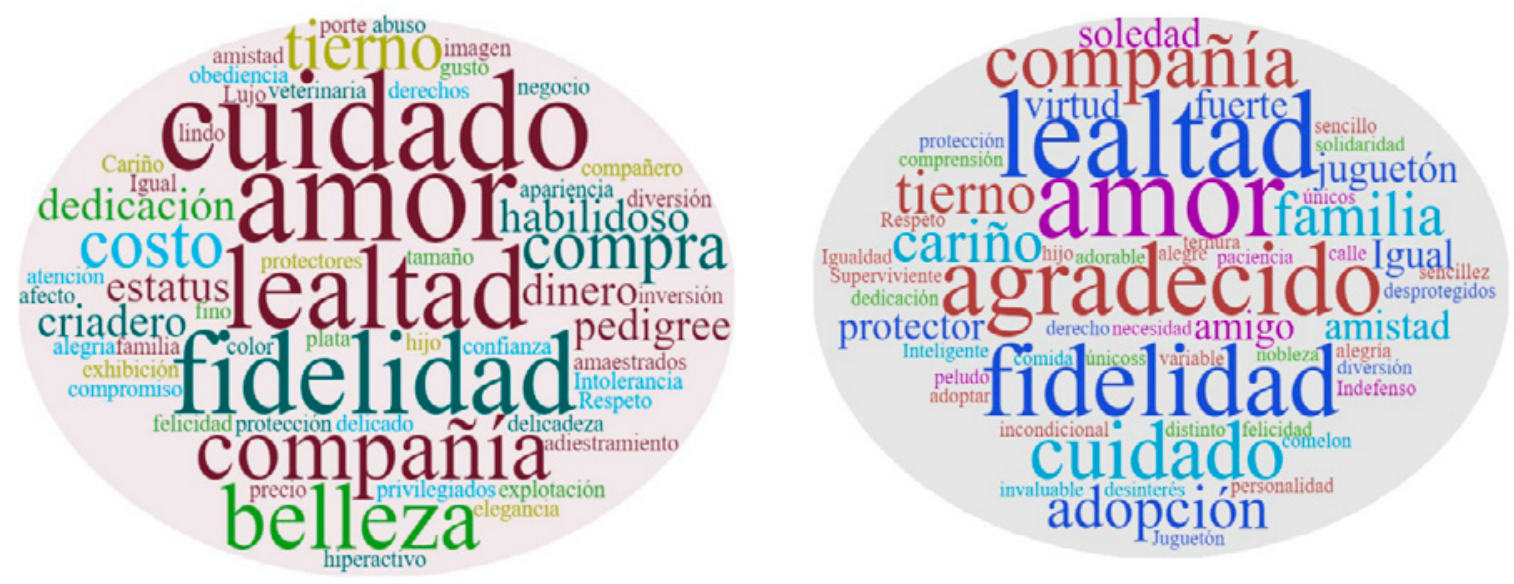

Figura 6.

Comparación de las palabras más frecuentes para describir a los perros de raza y criollos.

Nota. A la izquierda se observan las descripciones para los perros de raza, y a la derecha, las de perros criollos. 
Además, en cuanto al análisis de los atributos de los perros, se identificaron diferencias estadísticamente significativas en las siguientes afirmaciones: el perro de raza/ criollo "es una compañía para los seres humanos", "pasa desapercibido entre las personas", "requiere cuidado especial", "come de todo" y "requiere cuidado estético especial y se reconoce como todo terreno" (véase Tabla 1).

En cuanto a las prácticas de consumo, las diferencias estadísticamente significativas se observaron en las afirmaciones: "el tipo de perro que una persona tiene es símbolo de éxito y prestigio" y "las personas que tienen perros son más atractivas que otras que no tienen". Las afirmaciones como "algunas personas se sienten atraídas por adquirir perros costosos", "a algunas personas les gusta ser dueñas de animales domésticos que impresionan a los demás" y "algunas personas compran perros de raza para llamar la atención" muestran diferencias moderadas, según se puede observar en la Tabla 2.

Tabla 1.

Diferencias de medias de las puntuaciones entre las representaciones sociales asociadas a los perros de raza y criollos

\begin{tabular}{|c|c|c|c|c|c|c|c|}
\hline \multirow{2}{*}{ Factores } & \multirow[t]{2}{*}{$t$} & \multirow[t]{2}{*}{$g l$} & \multirow[t]{2}{*}{ sig. } & \multicolumn{2}{|c|}{ Raza } & \multicolumn{2}{|c|}{ Criollo } \\
\hline & & & & Media & D. $E$. & Media & D. E. \\
\hline \multicolumn{8}{|l|}{ Factores afectivos } \\
\hline Es compañía para los seres humanos & -3.12 & 77 & $<.01$ & 4.73 & 0.59 & 4.94 & 0.31 \\
\hline Es integrante de la familia & -1.21 & 77 & .22 & 4.73 & 0.76 & 4.84 & 0.68 \\
\hline \multicolumn{8}{|l|}{ Factores sociales } \\
\hline Pasa desapercibido entre las personas & -6.76 & 77 & $<.01$ & 2.07 & 1.19 & 3.39 & 1.32 \\
\hline Le da estatus a su dueño & 0.67 & 77 & $<.50$ & 2.70 & 1.65 & 2.57 & 1.30 \\
\hline \multicolumn{8}{|l|}{ Factores de cuidado } \\
\hline Requiere un cuidado especial & 2.53 & 77 & $<.05$ & 4.12 & 1.24 & 3.66 & 1.40 \\
\hline Come de todo & -8.97 & 77 & $<.01$ & 2.35 & 1.32 & 3.88 & 1.24 \\
\hline Requiere cuidado estético & 6.23 & 77 & $<.01$ & 3.79 & 1.27 & 2.67 & 1.26 \\
\hline \multicolumn{8}{|l|}{ Factores de comportamiento y fuerza } \\
\hline Se reconoce como todo terreno & 1.35 & 77 & $<.01$ & 2.33 & 1.28 & 4.47 & 0.96 \\
\hline Tiene una personalidad definida & 1.86 & 77 & .06 & 3.92 & 1.40 & 4.21 & 1.21 \\
\hline
\end{tabular}

Tabla 2.

Diferencias de medias entre los propietarios de perros criollos y los de raza para los items de prácticas de consumo conspicuo en la tenencia de una mascota

\section{Gusto mascotas}

Algunas personas se sienten atraídas por adquirir perros costosos

La gente compra perros porque le gusta el lujo en su vida

Las personas que compran perros con características raras lo hacen porque son más caros que otros perros

Exhibición

A algunas personas les gusta ser dueñas de animales domésticos que impresionan a los demás

Algunas personas compran perros para presumir

Algunas personas compran perros de raza para llamar la atención

Demostración social

Algunas personas compran perros para mejorar su imagen ante los demás El tipo de perro que una persona tiene es símbolo de éxito y prestigio

Algunas personas pagarían más por un perro que les genere estatus

Identificación con un grupo

Las personas quieren tener los mismos perros de sus amigos

Las personas que tienen perros son más atractivas que otras que no tienen

Algunas personas consideran que tener perros llamativos da popularidad entre amigos y colegas

(1)


150

Factores que motivan la compra de productos de lujo

Finalmente, en la etapa cuantitativa se indagó acerca de productos y servicios de lujo que los propietarios adquieren para sus mascotas, por medio del análisis de las frecuencias de los productos mencionados por los participantes. Para este paso, se discriminaron los hallazgos en función del tipo de perro - raza o criollo-, donde el $9 \%$ de la muestra correspondiente a los propietarios de perros criollos manifestó adquirir productos de lujo para su mascota - ropa y accesorios-, mientras que en los propietarios de los perros de raza se encontró que el $22 \%$ adquiere productos de lujo, también con productos como ropa y accesorios, aunque incluidos algunos poco inusuales, como coches.

Por otra parte, para el análisis de los datos textuales, se tomó un corpus de preguntas abiertas, $\mathrm{y}$, de acuerdo con el análisis de coocurrencias - que se puede observar en la Figura 7-, se encontró que ocho nodos describen las conceptualizaciones de los participantes acerca del consumo de productos o servicios de lujo para sus perros. En particular, el clúster de mayor frecuencia - en color azul turquesapodría denominarse como "función de la compra de productos de lujo", donde resaltan palabras como "querer dar gusto", "consentir", "lucir", "dinero", entre otras. En dicho clúster, se evidencia una necesidad de satisfacer un gusto personal por mantener "lindos" a los perros, y que, a su vez, "ese gusto debe ser lucido". Por otra parte, el clúster de color rojo puede ser entendido como "el consumo de lujo es para los que tienen dinero", y en este resaltan palabras como "tener", "económico", "facilidades", "mascotas" y "comodidad", que se refieren a que en el caso en que las personas tienen comodidades u holgura económica, pueden permitirse el consumo de productos o servicios de lujo para sus mascotas.

Y por último, en intersección con el clúster de color rojo, se observa un clúster de color amarillo claro, en el que se habla de que "solo quienes pertenecen a un nivel socioeconómico alto pueden consumir artículos de lujo para sus perros"; igualmente, el clúster de color naranja, en el que habla de que si las personas "tienen la capacidad" y "les gusta algo", pueden "adquirirlo" porque tienen la capacidad para ello. Otros clústeres muestran la necesidad de adquirir productos que permitan presumir con los perros en redes sociales, de lucir los perros con un aspecto estético aceptable, de la necesidad de tener recursos económicos para poder consumir artículos de lujo, y, finalmente, de la necesidad de llamar la atención con el consumo de dichos productos o servicios de lujo.

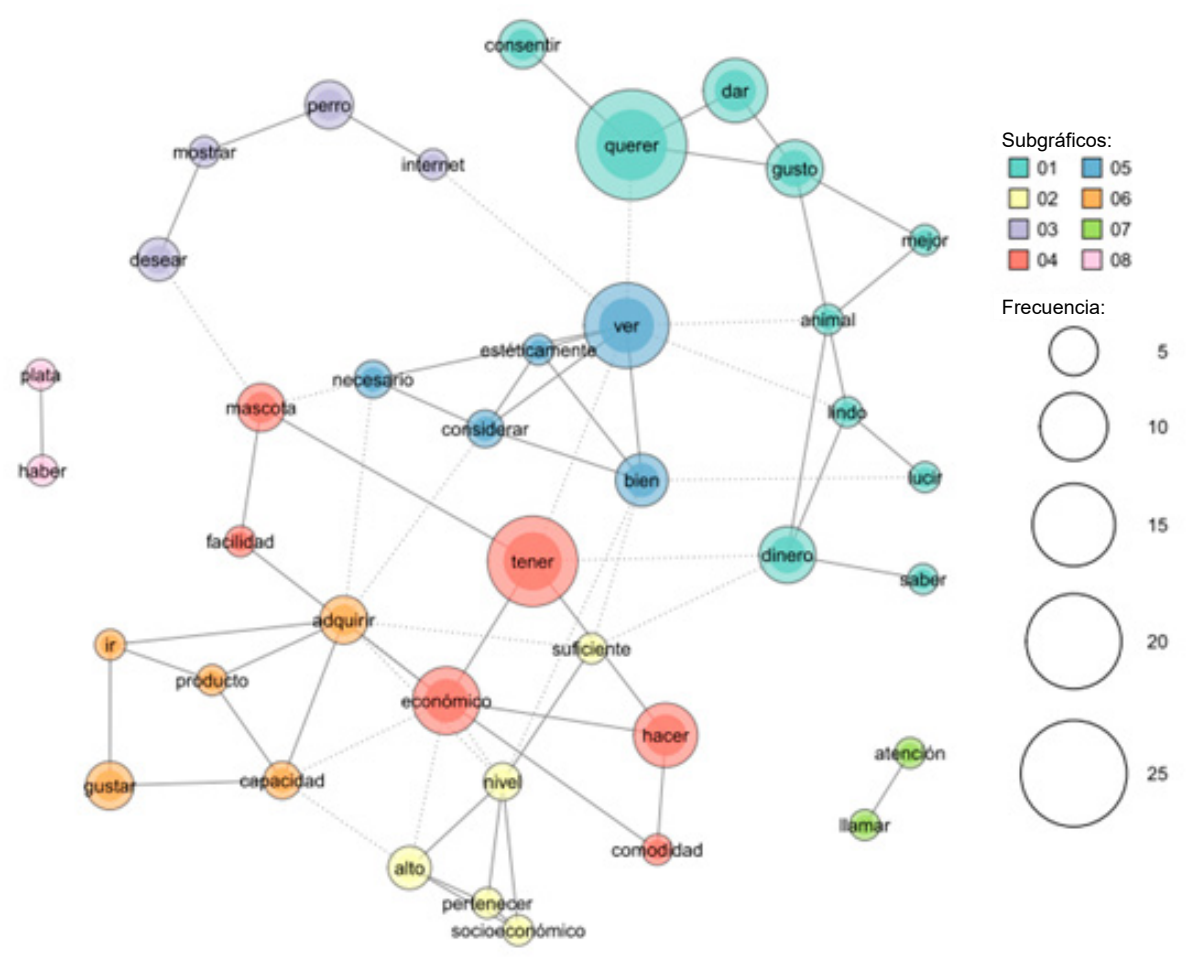

Figura 7.

Red de coocurrencias acerca de las conceptualizaciones del consumo conspicuo de productos o servicios para perros

Nota. Los círculos representan los nodos de las palabras más frecuentemente mencionadas. Los colores de los círculos representan el grado de afinidad semántica por clúster. La red está compuesta por 39 nodos, 68 conexiones y una densidad en la red de .092. Se utilizó un filtro jaccard (top 60), y las unidades de análisis fueron las oraciones. 


\section{Discusión}

Como se planteó en el objetivo de investigación, este estudio permitió reconocer aspectos representativos de las mascotas - perros criollos o de raza - y los significados sociales atribuidos a ellas. Anivel general, se encontró que el perro de raza se caracteriza como aquel que requiere un "cuidado especial", que necesita "cuidados estéticos" y que tiene una "personalidad definida" por la raza, mientras que el perro criollo fue reconocido como un perro "todo terreno", con una "personalidad definida", pero no determinada por sus características genéticas, y como una mascota que pasa desapercibida entre las personas, a diferencia del perro de raza.

Asimismo, los resultados muestran la expresión "amor" como aquella de mayor relevancia entre los participantes, al definir a ambos tipos de perros. Adicionalmente, se pueden observar las palabras "lealtad" y "fidelidad" en ambos grupos, y también expresiones como "compañía", "cuidado", "responsabilidad", "familia", "amigo" y "amistad", lo cual demuestra que los significados de las representaciones sociales construidas en torno a los perros son el resultado de la relación entre el objeto - perros-y los individuos - propietarios de mascotas - (Navarro \& Restrepo, 2013), que son tan cercanas que pueden llevar a las personas, incluso, al punto de considerar a una mascota como parte de la familia.

Un segundo elemento por resaltar es la construcción del significado que se le atribuye al hecho de tener un perro de raza o uno criollo - que en algunos casos se denomina raza única-, puesto que, como se observa en la descripción de resultados, en principio se indagaba por la categoría de perros en general, pero posteriormente se hablaba de perros criollos y luego de los de raza. Lo que llama la atención es que, cuando se menciona a los perros en general, las personas hablan de aspectos afectivos principalmente, pero luego, al ir agregando la clasificación —raza o criollo-, comienzan a aparecer elementos que cambian los significados de dichos animales.

Aun cuando las razas de los perros han sido una intervención humana con el propósito de seleccionar rasgos para el apoyo a las labores - en un primer lugar-, en la actualidad los perros han trascendido a los espacios familiares, y se han convertido en integrantes de la familia, en "hijos", en compañeros, en amigos, y en seres que requieren una asignación presupuestal mensual para las satisfacción tanto de sus necesidades básicas - comida y cuidado de saludcomo de actividades de bienestar - entrenamiento, paseo, diversión y ejercicio- y objetos suntuarios - collares, ropa, zapatos, peinados, carpas protectoras de lluvia, etc.- .
Respecto a esto último, se identificó que el consumo conspicuo se da tanto para la tenencia de perros de raza como en perros criollos, porque el lujo y el estatus están ligados a los significados que las personas construyen y que están enmarcados en un contexto sociocultural, como se ha observado en estudios previamente citados, en donde la percepción de lo que es y no es un objeto de lujo - en el caso del presente estudio, los perros- puede depender del contexto y de las personas involucradas.

Así, respecto a las representaciones sociales específicamente de los perros criollos, esta investigación permitió identificar un factor que se reconoce a quienes son propietarios de dichos perros, y es su generosidad y compromiso al realizar las adopciones, pues estas prácticas se convierten en una forma de ganar reconocimiento social y, en cierta medida, de generar formas de autoridad moral en comparación con quienes adquieren sus perros en intercambios monetarios.

De este modo, dichas personas reconocen valores afectivos en sus animales, construyen vínculos con ellos y les asignan roles especiales adaptados a las necesidades humanas; roles que van más allá del apoyo en el trabajo - como fuera en algún momento la filosofía de creación de las razas-, pues hoy día los perros no solo realizan trabajos de pastoreo, caza, seguridad, entre otros, sino que se han convertido en miembros de las familias (Martinez, 2019). Esto coincide con los hallazgos de Hirschman (1994), quien observó que los animales sirven a sus dueños actuando como compañeros, "hijos" o sustitutos de afecto.

Por otra parte, uno de los hallazgos más interesantes tiene que ver con las representaciones sociales respecto a los "perros de raza" y las prácticas de consumo conspicuo que se reconocen en propietarios de estas mascotas, pues dicha representación social se construye con los significados centrales de "amor", "cuidado" y "fidelidad", seguidos por algunas expresiones relevantes en el sistema periférico, como lo son "lealtad", "compañía", "belleza", "amigo", "compra" y "costo". Estas expresiones nos permiten ver que, además de los aspectos afectivos relacionados con la interacción entre perros y humanos, quienes tienen perros de raza les han dotado de otros roles y significados en función de su vida cotidiana, como pasa con el cuidado, el costo y la belleza, expresiones que marcan diferencias claras en las representaciones sociales acerca de los perros de raza y los criollos. En este contexto, y a la luz de los hallazgos de Hirschman (1994), se reconoce un segundo rol en la relación humano-animal, en la que estos se ven como objetos y productos.

Adicionalmente, en dicha relación se observa un valor social, entendido como aquella necesidad de estatus que los propietarios buscan satisfacer por medio de las mascotas. 
152

En esta investigación, las expresiones identificadas en el sistema periférico ubican a los perros de raza en condición de objetos, puesto que se pueden comprar y porque se les atribuye valor estético, como también los reportó Holbrook y Woodside (2008). Por lo tanto, se identifica que la tenencia de perros de raza está orientada a gustos personales y afectivos, y que, a su vez, está relacionada con el cumplimiento de logros personales, como alcanzar estatus, ejercer actividades de demostración social, e incluso compartir experiencias en torno a la raza de la mascota, con lo cual se adquiere, además, una identidad a través de las características del perro; es por ello que la raza de la mascota es muy relevante y se considera un aspecto diferencial a la hora de conformar estos grupos sociales.

De esta manera, los hallazgos confirman que los valores propuestos por Vigneron y Johnson (1999) se aplican a los significados desarrollados para los perros de raza y criollos, pues se asocian a valores sociales familiares y de grupos de propietarios, así como a valores económicos, de calidad, hedónicos y afectivos, relacionados con el ocio, las actividades de esparcimiento y las ocasiones lúdicas. En este último punto, desde la perspectiva de Minjung y Back (2020), la escasez de tiempo puede interpretarse como un factor importante en el desarrollo de la percepción de lujo, de manera que el tiempo de ocio con las mascotas puede comenzar a ser considerado una práctica de consumo conspicuo. De hecho, es de destacar que los clústeres identificados en esta investigación son afines con los valores de lujo simbólico, de experiencia y funcionales, acorde con los hallazgos de Zhang y Haidong (2019).

Finalmente, se sugiere para próximas investigaciones aplicar el estudio de representaciones sociales en espacios rurales para hacer un comparativo entre lo urbano y lo rural, y en población de adultos mayores y pacientes asistidos terapéuticamente con la ayuda de perros. Asimismo, resulta importante analizar cómo se desarrollan estos procesos simbólicos a través de los entornos digitales, en los cuales la visibilidad y ostentación alrededor de mascotas, posesiones y estilos de vida ocupan las dinámicas de las redes sociales (Rauzzino \& Correa, 2017). En relación con el consumo conspicuo, se sugiere profundizar en la identidad que los jóvenes adquieren cuando están con sus pares sociales dueños también de mascotas.

\section{Referencias}

Abric, J. (2001). Prácticas sociales y representaciones. Coyoacán.

Ballestas, L. (2018, junio 12). Tres billones de pesos, el gasto de las familias en sus mascotas. Periódico El Tiempo. https://www.eltiempo.com/economia/finanzas-personales/ dinero-que-invierten-las-familias-colombianas-en-el-cui dado-de-sus-mascotas-228908

Barrera, G., Giamal, Y., Mustaca, A., \& Bentosela, M. (2012). Relación entre el tipo de alojamiento y las respuestas de mirada, sociabilidad y miedo-apaciguamiento en perros. Suma Psicológica, 19(2), 7-18. https://dialnet.unirioja.es/descar ga/articulo/4232412.pdf

Blasco-Gil, Y., González, L., Pavón-Romero, A., MercadoEstrada, M., Pavón-Romero, C., Cabrera, A., GarzónFarinós, F., \& Peset, F. (2020). Enriqueciendo la investigación en humanidades digitales. Análisis de textos de claustros académicos de la Universidad de Valencia (1775-1779) con KH Coder. Revista española de Documentación Científica, 43(1), e257. http://dx.doi.org/10.3989/redc.2020.S1

Cabella, W., \& Nathan, M. (2018). Los desafios de la baja fecundidad en América Latina y el Caribe. UNFPA. https:// lac.unfpa.org/sites/default/files/pub-pdf/Baja\%20fecun didad $\% 20$ en $\% 20$ ALC $\% 20-\% 20$ version $\% 20$ web $\% 20$ espa $\%$ C3\%B1ol.pdf

Camilleri, L., Gill, P., \& Jago, A. (2020). The role of moral disengagement and animal empathy in the meat paradox. Personality and Individual Differences, 164(1), 110103. https://doi.org/10.1016/j.paid.2020.110103

Cekavicius, T., \& Pajarskaite, M. (2012). Pets as Status Symbols (Tesis de Maestría). Jonkoping University, EE. UU. https://www.diva-portal.org/smash/get/diva2:531243/FU LLTEXT02.pdf

Coll, C., \& De La Rosa, S. (2018). Comportamiento de compra del consumidor de productos para mascotas en Latinoamérica. Adgnosis, 7(7), 29-48. https://doi. org/10.21803/adgnosis.v7i7.291

Figueras, A., \& Morero, H. (2013). La teoría del consumo y de los ciclos en Thorstein Veblen. Revista De Economía Institucional, 15(28). https://revistas.uexternado.edu.co/in dex.php/ecoins/article/view/3465/3352

Folgueiras-Bertomeu, P., \& Ramirez, C. (2017). Elaboración de técnicas de recogida de información en diseños mixtos. Un ejemplo de estudio en aprendizaje-servicio. REIRE Revista d'Innovació i Recerca en Educació, 10(2), 64-78. https:// doi.org/10.1344/reire2017.10.218069

Higuchi, K. (2016). KH Coder 3 Reference Manual. Ritsumeikan University. http://khc.sourceforge.net/en/man ual en v3.pdf

Hirschman, E. (1994). Consumers and Their Animal Companions. Journal of Consumer Research, 20(4), 616632. https://doi.org/10.1086/209374

Holbrook, M., \& Woodside, A. (2008). Animal companions, consumption experiences, and the marketing of pets: Transcending boundaries in the animal-human distinction. Journal of Business Research, 61(5), 377-381. https://doi. org/10.1016/j.jbusres.2007.06.024

Jakovcevic, A., Irrazábal, M., \& Bentosela, M. (2011). Cognición social en animales y humanos: ¿es posible 
establecer un Continuo? Suma Psicológica, 18(1), 35-46. http://www.scielo.org.co/pdf/sumps/v18n1/v18n1a04.pdf

Kiattipoom, K., \& Heesup, H. (2019). What drives customers' willingness to pay price premiums for luxury gastronomic experiences at michelin-starred restaurants? International Journal of Hospitality Management, 82, 209-219. https:// doi.org/10.1016/j.ijhm.2019.04.024

Kirk, C. (2019). Dogs have masters, cats have staff: Consumers' psychological ownership and their economic valuation of pets. Journal of Business Research, 99, 306-318. https:// doi.org/10.1016/j.jbusres.2019.02.057

Martinez, J. (2019, junio 27). La economía alrededor de las mascotas en Bogotá. Observatorio de Desarrollo Económico. http://observatorio.desarrolloeconomico.gov.co/comercioal-por-menor-industria-servicios/la-economia-alrededor -de-las-mascotas-en-bogota

Molina, J., Moreno, J. H., \& Vásquez, H. (2010). Análisis referencial de las representaciones sociales sobre la violencia doméstica. Acta Colombiana de Psicología, 13(2), 129-148. https://actacolombianapsicologia.ucatolica.edu.co/article/ view/375

Materán, A. (2008). Las representaciones sociales: un referente teórico para la investigación educativa. Geoenseñanza, 13, 243-248.http://www.redalyc.org/articulo.oa? id=360212300 10

Minjung, S., \& Back, K-J. (2020). The luxury of doing nothing: inferring luxury from idleness display in travel setting. Journal of Travel \& Tourism Marketing, 37, 409-417. https://doi.org/10.1080/10548408.2020.1783428

Moscovici, S. (1985). Psicología social. Paidós.

Mozzocco, P., Rucker, D., Galinsky, A., \& Anderson, E. (2012). Direct and vicarious conspicuous consumption: Identification with low-status groups increases the desire for high-status goods. Journal of Consumer Psychology, 22, 520-528. https://doi.org/10.1016/j.jcps.2012.07.002

Navarro, O., \& Restrepo, D. (2013). Representaciones sociales: perspectivas teóricas y metodológicas. Revista CES Psicología, 6(1), i-iv. https://revistas.ces.edu.co/index.php/ psicologia/article/view/2617

Onofre-Chaves, D. (2017). La investigación en estilos de vida en psicología de consumidor. Cultura, Educación $y$ Sociedad, 8(1), 51-70. http://dx.doi.org/10.17981/ cultedusoc.8.1.2017.04

Otterbring, T., Sundie, J., Yexin , J., \& Hille, S. (2020). Evolutionary psychological consumer research: Bold, bright, but better with behavior. Journal or Business Research, 120, 473-484. https://doi.org/10.1016/j. jbusres.2020.07.010
Power, E. (2011). Furry families: making a human-dog family through home. Journal Social \& Cultural Geography, 9, 535-555. https://doi.org/10.1080/14649360802217790

Quiroz, A. (2004). Actitudes y representaciones. Temas actuales de psicología social. Benemérita Universidad Autónoma de Puebla.

Rauzzino, A., \& Correa, J. C. (2017). Millennials sex differences on Snapchat perceived privacy. Suma Psicológica, 24(2), 129-134. https://doi.org/10.1016/j.sumpsi.2017.08.002

Ruiz, J. (2018). El lujo ilustrado vs. El lujo hipermoderno: el asunto moral del lujo. Revista de estudios de ciencias sociales y humanidades, 39, 93-101. http://hdl.handle. net/10396/17228

Sandoval-Escobar, M., Medina-Arboleda, I., Ávila-Campos, J., \& Moreno-Hernández, L. (2018). Aprendizaje de la marca: aproximaciones conceptuales desde la psicología del consumidor. Suma de Negocios, 9(20), 138-144. http://dx.doi. org/10.14349/sumneg/2018.V9.N20.A8

Trigg, A. (2016). Veblen, Bourdieu, and Conspicuous Consumption. Journal of Economic Issues, 35, 99-115. https://doi.org/10.1080/00213624.2001.11506342

Veblen, T. (1899). Teoría de la clase ociosa. Alianza Editorial. https://www.omegalfa.es/titulos.php?letra=t

Vergara, M. (2008). La naturaleza de las representaciones sociales. Revista Latinoamericana de Ciencias Sociales, Niñez y Juventud, 6, 55-80. https://www.redalyc.org/ pdf/773/77360103.pdf

Vigneron, F., \& Johnson, L. (1999). A Review and a Conceptual Framework of Prestige-Seeking Consumer Behavior. Academy of Marketing Science Review, 1, 1-15. https:// search.proquest.com/scholarly-journals/review-conceptual-framework-prestige-seeking/docview/200920758/ se- 2 ? accountid $=45660$

Vigneron, F., \& Johnson, L. (2004). Measuring perceptions of brand luxury. Journal of Brand Management, 11, 484-506. https://doi.org/10.1057/palgrave.bm.2540194

World Animal Protection. (2018). Latinoamericanos: el 95\% ven a sus mascotas como hijos o parte de sus familias. https://www.worldanimalprotection.cr/noticias/latinoa mericanos-el-95-ven-sus-mascotas-como-hijos-o-parte-desus-familias

Zhang, L., \& Haidong, Z. (2019). Personal value vs. luxury value: What are Chinese luxury consumers shopping for when buying luxury fashion goods? Journal of Retailing and Consumer Services, 51, 62-71. https://doi.org/10.1016/j. jretconser.2019.05.027 